\title{
Real-World Analysis of Switching Patients with Schizophrenia from Oral Risperidone or Oral Paliperidone to Once-Monthly Paliperidone Palmitate
}

\author{
Charmi Patel ${ }^{1} \cdot$ Bruno Emond $^{2} \cdot$ Marie-Hélène Lafeuille $^{2} \cdot$ Aurélie Côté-Sergent $^{2} \cdot$ Patrick Lefebvre $^{2} \cdot$ Neeta Tandon $^{1}$. \\ Antoine C. El Khoury ${ }^{1}$
}

Published online: 30 November 2019

(C) The Author(s) 2019

\begin{abstract}
Purpose Reducing the dosing frequency of antipsychotics (APs) with long-acting injectables (LAIs) such as once-monthly paliperidone palmitate (PP1M) can improve adherence and clinical outcomes for schizophrenia patients. This US study compared physical and psychiatric comorbidity-related outcomes, AP adherence, healthcare resource utilization (HRU), and costs pre- and post-transition to PP1M among schizophrenia patients treated with oral risperidone/paliperidone pre-PP1M transition.

Methods Health insurance claims from the IQVIA ${ }^{\mathrm{TM}}$ PharMetrics Plus database (01/01/2012-07/31/2018) were used to identify adults with $\geq 2$ schizophrenia diagnoses, $\geq 1$ claim for PP1M, and $\geq 30$ days of treatment with oral risperidone/ paliperidone in the 60 days before the first PP1M claim (i.e., the index date). Comorbidity-related outcomes, adherence to APs (measured via the proportion of days covered [PDC]), all-cause per-patient-per-month (PPPM) HRU, and all-cause PPPM medical, pharmacy, and total costs (i.e., sum of medical and pharmacy costs) during the 6-month periods pre- and post-transition to PP1M were compared using generalized estimating equation models adjusted for repeated measurements. Analyses were replicated in the subset of patients with $\geq 1$ all-cause inpatient stay pre-PP1M transition.

Findings Among 427 schizophrenia patients transitioning from oral risperidone/paliperidone to PP1M, the mean age was 41.1 years and $37.9 \%$ were female. Following the PP1M transition, patients were less likely to have claims with a diagnosis for psychoses (odds ratio [OR] $0.41 ; P<0.001$ ), hypertension (OR 0.80; $P=0.011$ ), depression (OR $0.70 ; P<0.001$ ), drug abuse (OR 0.60; $P<0.001$ ), substance-related and addictive disorders (OR 0.73; $P=0.003$ ), bipolar and related disorders (OR $0.59 ; P<0.001$ ), sleep-wake disorders (OR 0.68; $P=0.017$ ), anxiety disorders (OR $0.78 ; P=0.034$ ), and other conditions that may require a focus of clinical attention (OR $0.58 ; P<0.001)$. Mean PDC by APs was higher post-PP1M $($ mean $=0.81)$ versus pre-PP1M (mean $=0.68)$ transition. Post-PP1M, patients were less likely to have an all-cause emergency room visit (OR $0.51 ; P<0.001)$ or inpatient stay (OR $0.39 ; P<0.001)$ compared to pre-PP1M. All-cause total healthcare costs remained similar post- versus pre-transition to PP1M (mean monthly cost difference $[\mathrm{MMCD}]=\$ 228 ; P=0.260$ ). Pharmacy costs increased post-PP1M (MMCD $=\$ 960 ; P<0.001)$, but were offset by decreasing medical costs $(\mathrm{MMCD}=-\$ 732$; $P<0.001)$, largely driven by lower costs related to inpatient stays $(\mathrm{MMCD}=-\$ 695 ; P<0.001)$ and emergency room visits (MMCD $=-\$ 63 ; P<0.001)$. For patients with $\geq 1$ all-cause inpatient stay pre-PP1M transition $(N=177)$, a more pronounced improvement in comorbidity-related outcomes, a more pronounced reduction in HRU, and a reduction in total healthcare costs $(\mathrm{MMCD}=-\$ 1308 ; P<0.001)$ were observed post-transition to PP1M.

Implications Among schizophrenia patients in the US, transitioning to PP1M following oral risperidone/paliperidone treatment was associated with improved comorbidity-related outcomes, higher adherence, and a reduction in HRU, while remaining cost neutral. Furthermore, patients with $\geq 1$ all-cause inpatient stay pre-PP1M transition had significantly lower total healthcare costs post-PP1M transition.
\end{abstract}

Extended author information available on the last page of the article 


\section{Key Points}

Switching patients from oral risperidone or oral paliperidone to once-monthly paliperidone palmitate was associated with improved comorbidity-related outcomes and higher adherence to antipsychotics.

Following the switch to once-monthly paliperidone palmitate, patients had fewer hospitalizations and emergency room visits, lower total medical costs, and similar total healthcare costs compared to the period prior to the switch.

For the subgroup of patients with $\geq 1$ all-cause inpatient stay prior to the switch, switching was associated with a reduction in total healthcare costs compared to the period prior to the switch.

\section{Introduction}

Schizophrenia is a remitting and relapsing psychiatric disorder associated with significant impairments of mental and social functioning, recurrent episodes of exacerbated psychotic symptoms, and a broad range of psychiatric and physical comorbidities [1].

The range of available treatments for schizophrenia has expanded over the last few decades with the introduction of oral atypical antipsychotics (OAA), which have become the standard of care owing to their diverse pharmacologic action and improved tolerability [2-4]. However, nonadherence to antipsychotics (APs) remains an issue in the treatment of schizophrenia. Up to two-thirds of commercially insured patients with schizophrenia in the United States (US) were found to have inadequate adherence to their AP medications $[5,6]$. Nonadherence hinders treatment success and can lead to poor outcomes and high risks of relapse and rehospitalization [7-11]. Nonadherence is also associated with increased healthcare resource utilization (HRU) and costs [11-13]. In a study published in 2008 and using data from the period 1997-2003, annual hospitalization costs associated with schizophrenia in the US were estimated to be 806 million US dollars (USD), of which 106 million USD were attributed to nonadherence [13].

Treatment strategies that optimize adherence to APs and minimize risk of relapse have the potential to optimize treatment effectiveness, improve overall patient clinical outcomes, and reduce healthcare costs [14]. The use of longacting injectables (LAIs) has been associated with improved medication adherence and significantly lower relapse and hospitalization rates in patients with schizophrenia $[15,16]$. Therefore, switching patients to LAIs could lead to better adherence and outcomes, as reported in a recent real-world study of Medicaid beneficiaries with schizophrenia [17].

PP1M is an LAI that was approved for acute maintenance and treatment of schizophrenia in July 2009, and for the treatment of schizoaffective disorder in November 2014 [18, 19]. The prescribing guidelines for PP1M recommend tolerability testing with oral risperidone or oral paliperidone prior to PP1M initiation for oral risperidone/paliperidonenaïve patients [20].

To date, most studies evaluating clinical and economic outcomes in Medicaid-insured and commercially insured patients treated with OAAs or PP1M used a cohort design to compare outcomes between the two groups [5, 21-23]. However, there is a need to better understand whether switching patients from OAAs such as risperidone or paliperidone to PP1M results in better clinical and economic outcomes post- versus pre-transition. This study compared physical and psychiatric comorbidity-related outcomes, adherence to APs, HRU, and healthcare costs pre- and post-transition to PP1M among patients with schizophrenia who were treated with oral risperidone or oral paliperidone prior to PP1M initiation in the US.

\section{Methods}

\subsection{Data Source}

Health insurance claims from IQVIA ${ }^{\mathrm{TM}}$ PharMetrics Plus database dating from $01 / 01 / 2012$ to $07 / 31 / 2018$ were analyzed. This database comprises more than 150 million unique enrollees across the US with commercial insurance, Medicare Advantage, managed Medicaid, self-insured, and pharmacyonly plans. Available information includes demographic characteristics (e.g., age, sex, and insurance plan and product type), inpatient and outpatient diagnoses and procedures, and pre-rebate costs paid by health plans to providers. Data were compliant with the patient confidentiality requirement of the Health Insurance Portability and Accountability Act (HIPAA). Per Title 45 of CFR, Part 46.101(b) (4) [24], the analysis of our study is exempt from institutional review for the following reasons: (1) it is a retrospective analysis of existing data (hence no patient intervention or interaction); and (2) no patient-identifiable information is included in the claims dataset.

\subsection{Study Design and Population}

This study used a retrospective observational cohort design. The index date was defined as the date of the first PP1M claim. Only patients with $\geq 30$ days of treatment with oral risperidone or oral paliperidone in the 60 days preceding the index date were included in the study. The baseline (i.e., pre-PP1M transition) and observation (i.e., post-PP1M 
transition) periods were defined as the 6-month periods preceding and following the index date, respectively.

To be included in the study, patients were required to have: $\geq 2$ claims with a diagnosis of schizophrenia (International Classification of Diseases, Ninth Revision [ICD9]: 295.XX; International Classification of Diseases, Tenth Revision [ICD-10]: F20.XX, F21, F25.X) during the continuous insurance eligibility period, including $\geq 1$ in the 2 years prior to the index date; $\geq 1$ claim for PP1M during the continuous insurance eligibility period; $\geq 30$ days of treatment on oral risperidone or oral paliperidone in the 60 days prior to the first PP1M claim; continuous insurance eligibility for $\geq 6$ months before and after the index date; and an age of $\geq 18$ years as of the index date. In a subgroup analysis of patients with $\geq 1$ all-cause hospitalization, patients were additionally required to have $\geq 1$ all-cause inpatient stay in the baseline period.

\subsection{Study Measures}

Demographic characteristics were described during the baseline period and included age, gender, US census region, insurance plan and product type, year of index date, and Quan-Charlson Comorbidity Index (Quan-CCI). The following measures were compared for patients pre- and posttransition to PP1M: (1) physical and psychiatric comorbidity-related outcomes for categories with the highest proportions of patients (i.e., $\geq 10 \%$ ) in the data, based on the Elixhauser algorithm [25] (which includes both physical and psychiatric comorbidities) and the Diagnostic and Statistical Manual of Mental Disorders-5th Edition (DSMV) [26]; (2) adherence to APs, as measured by the proportion of days covered (PDC) by APs and the proportion of patients with a $\mathrm{PDC} \geq 80 \%$; (3) all-cause per-patient-permonth (PPPM) HRU, which consisted of the proportion of patients with $\geq 1$ outpatient visit, $\geq 1$ emergency room visit, or $\geq 1$ inpatient stay, of the number of outpatient and emergency room visits, and of the number of inpatient stays and days spent in an inpatient setting; and (4) all-cause PPPM medical, pharmacy, and total healthcare costs (i.e., sum of pharmacy and medical costs). Medical costs included costs related to outpatient visits, emergency room visits, inpatient stays, and other services (i.e., durable medical equipment and dental and vision care). All cost outcomes were inflated to 2018 US dollars using the medical care component of the Consumer Price Index [27].

\subsection{Statistical Analysis}

Descriptive statistics were generated to summarize demographic and clinical characteristics. Means, medians, and standard deviations (SDs) were used for continuous variables, while counts and percentages were used for categorical variables. HRU and cost outcomes were reported PPPM. Physical and psychiatric comorbidity-related outcomes, adherence to APs, HRU, and costs during the 6-month periods pre- and post-transition to PP1M were compared using generalized estimating equation models adjusted for repeated measurements. For binary outcomes (i.e., comorbidities and adherence), a binomial distribution with a logit link was used to calculate odds ratios (ORs). For count variables (i.e., HRU outcomes), a Poisson distribution with a log link was used to calculate rate ratios (RRs), and for continuous outcomes (i.e., costs), a normal distribution with an identity link was used to calculate mean monthly cost differences (MMCDs). To account for the overdispersion of HRU outcomes and the nonnormal distribution of cost outcomes, nonparametric bootstrap procedures with 500 replications were used to calculate $95 \%$ confidence intervals (CIs) and $P$ values $(P)$ associated with RRs and MMCDs. All study measures were re-evaluated as part of a subgroup analysis that included only patients with $\geq 1$ all-cause inpatient stay during the baseline period.

\section{Results}

A total of 427 patients with schizophrenia who transitioned from oral risperidone or oral paliperidone to PP1M were included in the study (Fig. 1).

\subsection{Demographic and Clinical Characteristics Before Transition to PP1M}

Baseline demographic and clinical characteristics are shown in Table 1. Mean age was $41.1(\mathrm{SD}=13.9)$ years and $37.9 \%$ of the patients were female. The South (29.5\%), Midwest (36.3\%), and West (26.5\%) US census regions were well represented, but fewer patients (7.7\%) lived in the Northeast region. Most patients were covered by managed Medicaid (61.1\%) or Medicare Advantage (18.0\%), and the majority of patients had a health maintenance organization (HMO) insurance plan (80.1\%). Mean Quan-CCI was 0.79 $(\mathrm{SD}=1.32)$.

Of the 427 patients included in the main analyses, 177 (41.5\%) had $\geq 1$ all-cause inpatient stay during the 6-month pre-PP1M period and were included in the subgroup analyses. The mean age of these patients was $39.3(\mathrm{SD}=14.4)$ years, and $37.9 \%$ were female. The proportion of patients from the South, Midwest, West, and Northeast US census regions was $29.4 \%, 45.8 \%, 14.1 \%$, and $10.7 \%$, respectively. Mean Quan-CCI was higher than for all patients included in the main analyses (mean $=0.95 ; \mathrm{SD}=1.42$ ). Most patients were covered by managed Medicaid (51.4\%); 68.9\% of patients had a HMO insurance plan. 


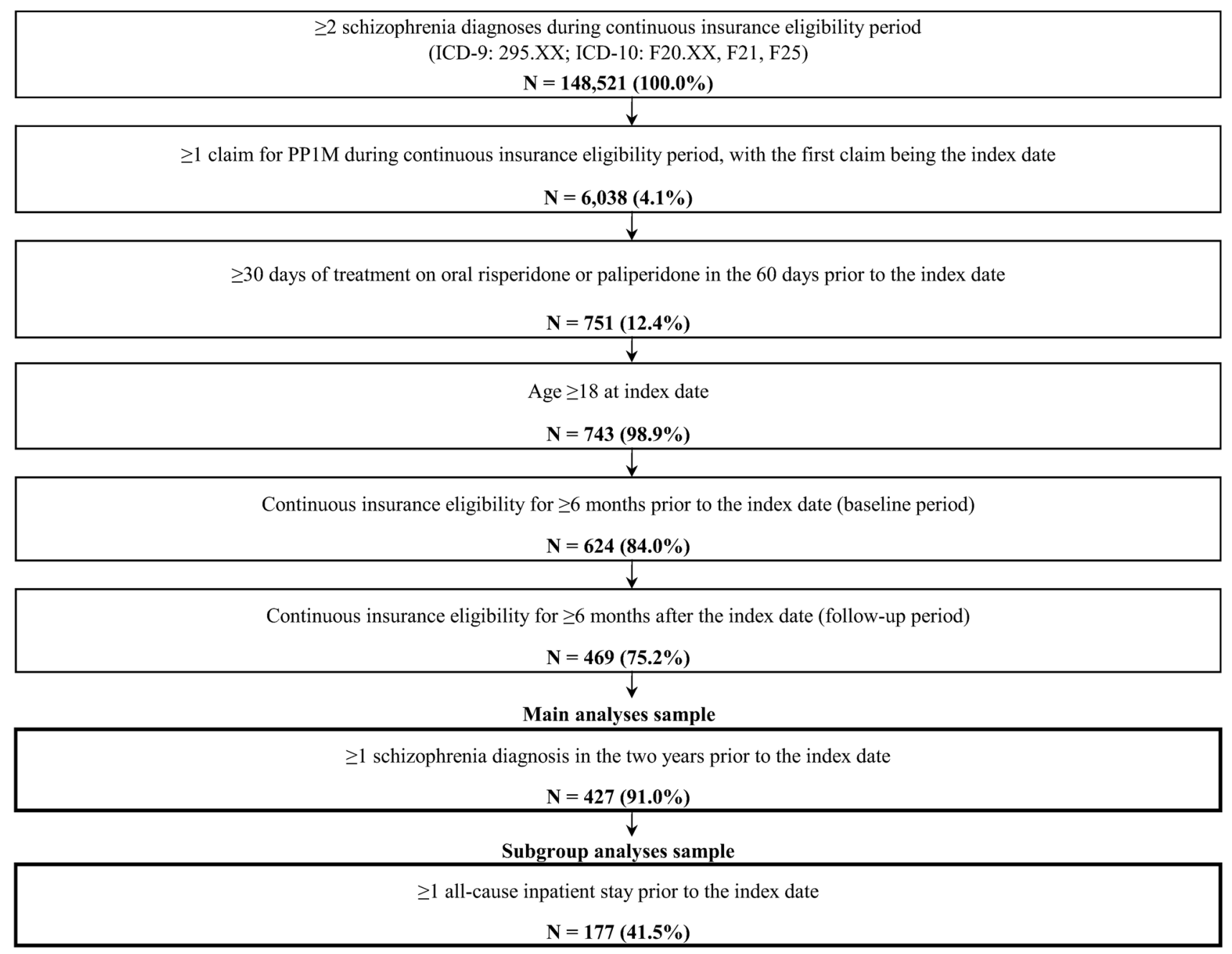

Fig. 1 Identification of the study population. ICD-9/10 International Classification of Diseases, Ninth/Tenth Revision, $P P 1 M$ once-monthly paliperidone palmitate

\subsection{Physical and Psychiatric Comorbidity-Related Outcomes Before and After Transition to PP1M}

The likelihood of having $\geq 1$ claim for each Elixhauser and DSM-V comorbidity pre- versus post-transition to PP1M is shown in Fig. 2. Following PP1M transition, and based on Elixhauser comorbidities, patients were 59\% less likely to have $\geq 1$ claim with a diagnosis for psychoses (OR $0.41, P<0.001$ ), 20\% less likely to have $\geq 1$ claim with a diagnosis for hypertension (OR 0.80, $P=0.011$ ), $30 \%$ less likely to have $\geq 1$ claim with a diagnosis for depression (OR $0.70, P<0.001$ ), and $40 \%$ less likely to have $\geq 1$ claim with a diagnosis for drug abuse (OR 0.60 , $P<0.001)$. Based on DSM-V comorbidities, patients were $27 \%, 41 \%, 32 \%, 22 \%$, and $42 \%$ less likely to have $\geq 1$ claim with a diagnosis for substance-related and addictive disorders (OR 0.73, $P=0.003$ ), bipolar and related disorders (OR 0.59, $P<0.001$ ), sleep-wake disorders (OR $0.68, P=0.017$ ), anxiety disorders (OR $0.78, P=0.034$ ), and other conditions that may require a focus of clinical attention (e.g., relation problems and abuse and neglect; OR $0.58, P<0.001$ ) following PP1M transition, respectively. Patients included in the subgroup with $\geq 1$ all-cause inpatient stay in the pre-PP1M period were less likely to have $\geq 1$ claim with a diagnosis for hypertension (OR 0.68 , $P=0.005$ ), obesity (OR $0.57, P=0.003$ ), depression (OR $0.55, P \leq 0.001$ ), drug abuse (OR 0.41, $P<0.001$ ), chronic pulmonary disease (OR $0.63, P=0.006$ ), substance-related and addictive disorders (OR $0.39, P<0.001)$, bipolar and related disorders (OR $0.46, P<0.001$ ), sleep-wake disorders (OR 0.49, $P=0.008$ ), anxiety disorders (OR 0.59, $P=0.002)$, and other conditions that may require a focus of clinical attention (OR $0.26, P<0.001$ ) following transition to PP1M. 
Table 1 Demographic and clinical characteristics evaluated during the 6-month period before transition to PP1M

\begin{tabular}{|c|c|c|}
\hline & All patients & $\begin{array}{l}\text { Subgroup analysis using patients } \\
\text { with } \geq 1 \text { baseline all-cause inpatient } \\
\text { stay }\end{array}$ \\
\hline & $N=427$ & $N=177$ \\
\hline Age at index date (years), mean \pm SD [median] & $41.1 \pm 13.9[41.0]$ & $39.3 \pm 14.4[38.0]$ \\
\hline \multicolumn{3}{|l|}{ Age categories, $n(\%)$} \\
\hline $18-24$ & $62(14.5)$ & $34(19.2)$ \\
\hline $25-34$ & $98(23.0)$ & $45(25.4)$ \\
\hline $35-44$ & $87(20.4)$ & $32(18.1)$ \\
\hline $45-54$ & $94(22.0)$ & $32(18.1)$ \\
\hline$>55$ & $86(20.1)$ & $34(19.2)$ \\
\hline Female, $n(\%)$ & $162(37.9)$ & $67(37.9)$ \\
\hline \multicolumn{3}{|l|}{ Region characteristics, ${ }^{\mathrm{a}} n(\%)$} \\
\hline South & $126(29.5)$ & $52(29.4)$ \\
\hline Midwest & $155(36.3)$ & $81(45.8)$ \\
\hline West & $113(26.5)$ & $25(14.1)$ \\
\hline Northeast & $33(7.7)$ & $19(10.7)$ \\
\hline \multicolumn{3}{|l|}{ Plan type, ${ }^{\mathrm{a}} n(\%)$} \\
\hline Managed Medicaid & $261(61.1)$ & $91(51.4)$ \\
\hline Medicare Advantage & $77(18.0)$ & $26(14.7)$ \\
\hline Commercial insurance & $50(11.7)$ & $32(18.1)$ \\
\hline Self-Insured & $37(8.7)$ & $27(15.3)$ \\
\hline Unknown/Missing & $2(0.5)$ & $1(0.6)$ \\
\hline \multicolumn{3}{|l|}{ Product type, ${ }^{\mathrm{a}} n(\%)$} \\
\hline Health maintenance organization & $342(80.1)$ & $122(68.9)$ \\
\hline Preferred provider organization & $73(17.1)$ & $49(27.7)$ \\
\hline Indemnity/traditional & $3(0.7)$ & $3(1.7)$ \\
\hline Point of service & $3(0.7)$ & $1(0.6)$ \\
\hline Consumer-directed health care & $4(0.9)$ & $1(0.6)$ \\
\hline Unknown/missing & $2(0.5)$ & $1(0.6)$ \\
\hline \multicolumn{3}{|l|}{ Year of index date, $n(\%)$} \\
\hline 2012 & $14(3.3)$ & $8(4.5)$ \\
\hline 2013 & $52(12.2)$ & $26(14.7)$ \\
\hline 2014 & $56(13.1)$ & $26(14.7)$ \\
\hline 2015 & $120(28.1)$ & $44(24.9)$ \\
\hline 2016 & $167(39.1)$ & $60(33.9)$ \\
\hline 2017 & $17(4.0)$ & $13(7.3)$ \\
\hline 2018 & $1(0.2)$ & $0(0.0)$ \\
\hline Quan-CCI, mean \pm SD [median] & $0.79 \pm 1.32[0.00]$ & $0.95 \pm 1.42[0.00]$ \\
\hline
\end{tabular}

$C C I$ Charlson Comorbiditylindex, $P P 1 M$ once-monthly paliperidone palmitate, $S D$ standard deviation

${ }^{a}$ As of month of index date

\subsection{Adherence Before and After Transition to PP1M}

Compared to the 6-month period pre-PP1M transition, adherence to APs improved following transition. Mean PDC was higher post-transition (mean $=0.81, \mathrm{SD}=0.22$ ) versus pretransition (mean $=0.68, \mathrm{SD}=0.25$; mean difference $=0.13$, $P<0.001$ ). For patients included in the subgroup with $\geq 1$ all-cause inpatient stay in the baseline period, mean PDC was also higher post-transition ( mean $=0.78, \mathrm{SD}=0.23$ ) versus pre-transition (mean $=0.64, \mathrm{SD}=0.26$; mean difference $=0.15, P<0.001)$. The proportion of patients with a PDC $\geq 80 \%$ significantly increased from 45.0 to $68.1 \%$ following PP1M transition (OR 2.62, $P<0.001$ ). Results were similar for patients with $\geq 1$ baseline all-cause inpatient stay, with the proportion of patients with a PDC $\geq 80 \%$ increasing from 37.9 to $61.6 \%$ (OR 2.63, $P<0.001$ ). 


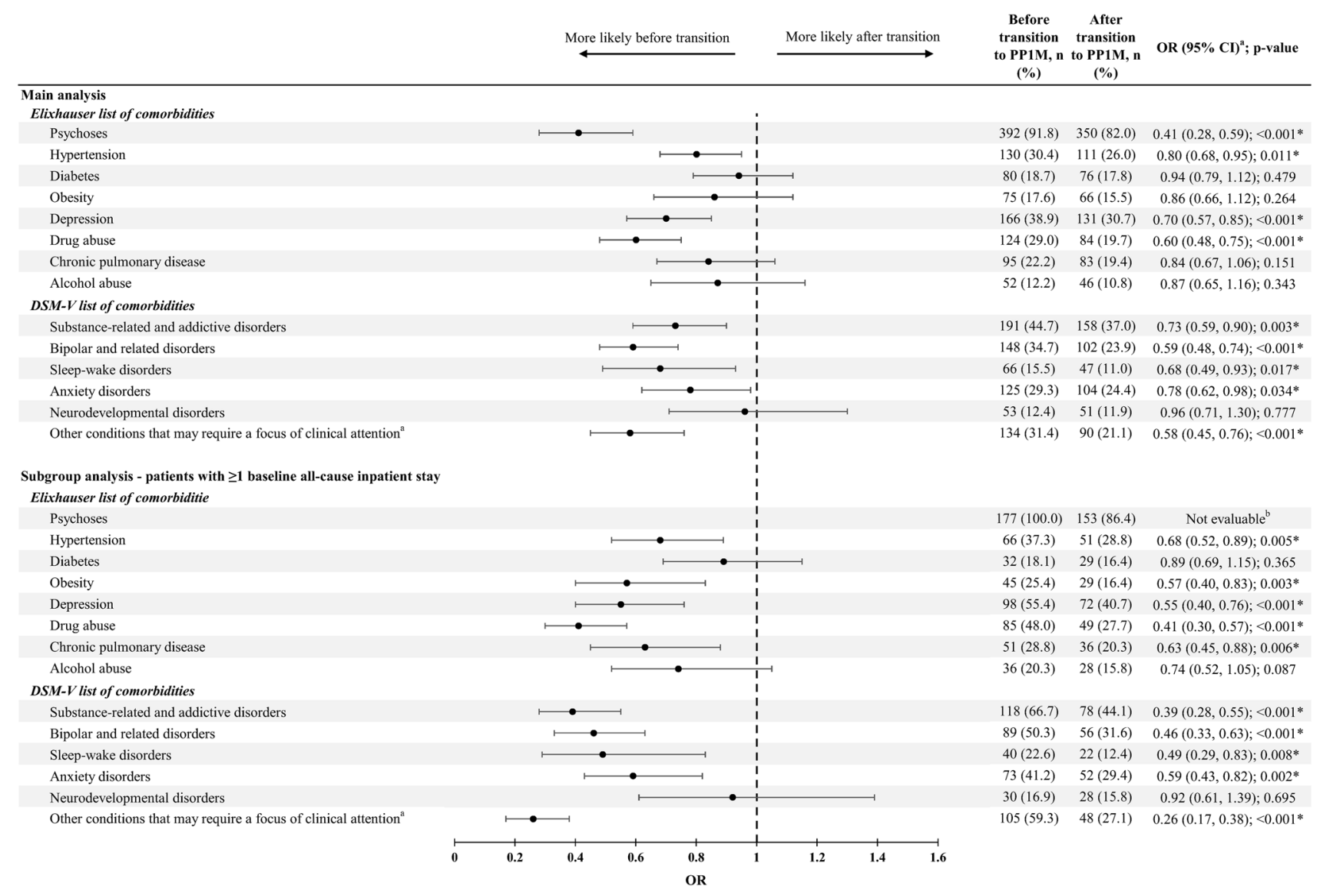

Fig. 2 Comparison of physical and psychiatric comorbidity-related outcomes 6 months before and after the transition to PP1M. CI confidence interval, DSM-V Diagnostic and Statistical Manual of Mental Disorders, 5th Edition, OR odds ratio, PPIM once-monthly paliperidone palmitate. ${ }^{*}$ Statistically significant $(P<0.05)$. ${ }^{\text {a }}$ These conditions refer to other conditions and problems that may be a focus of clinical attention or that may otherwise affect the diagnosis, course, prog-

\subsection{HRU and Costs Before and After Transition to PP1M}

Figure 3 shows likelihood and frequency data for all-cause PPPM HRU outcomes pre- versus post-transition to PP1M. Post-transition, patients were less likely to have an all-cause emergency room visit (OR $0.51, P<0.001$ ) or inpatient stay (OR 0.39, $P<0.001$ ) compared to the period pre-PP1M transition. Patients also had a fewer number of emergency room visits (RR 0.68, $P<0.001$ ), inpatient stays (RR 0.46, $P<0.001$ ), and days spent in an inpatient setting (RR 0.47 , $P<0.001)$ per month after the transition. Patients had a similar number of mental-health-related outpatient visits per month before and after the transition to PP1M (mean number of visits $=2.16$ versus 2.34; RR 1.08, $P=0.248$ ).

Figure 4 shows PPPM cost outcomes pre- versus posttransition to PP1M. All-cause total healthcare costs remained similar post- versus pre-transition to $\mathrm{PP} 1 \mathrm{M}(\mathrm{MMCD}=\$ 228$, nosis, or treatment of a patient's mental disorder. These conditions include relation problems, abuse and neglect, educational and occupational problems, housing and economic problems, other problems related to the social environment, other health service encounters for counseling and medical advice, and other circumstances of personal history. ${ }^{\mathrm{b}}$ The OR for psychoses is not evaluable since all patients had a diagnosis for psychoses in the pre-PP1M transition period

$P=0.260)$. All-cause pharmacy costs increased post-PP1M (MMCD $=\$ 960, P<0.001)$, but were offset by decreasing all-cause medical costs $(\mathrm{MMCD}=-\$ 732, P<0.001)$. The reduction in medical costs was mainly driven by lower costs related to inpatient stays (MMCD $=-\$ 695$, $P<0.001)$, followed by costs related to emergency room visits $(\mathrm{MMCD}=-\$ 63, P<0.001)$.

Patients included in the subgroup with $\geq 1$ all-cause inpatient stay in the baseline period were also less likely to have emergency room visits (OR 0.36, $P<0.001)$ post-PP1M, and had a fewer number of emergency room visits (RR 0.63, $P<0.001$ ), inpatient stays (RR $0.33, P<0.001$ ), and days spent in an inpatient setting (RR 0.34, $P<0.001)$ per month (Fig. 3). All-cause total healthcare costs were significantly lower following transition $(\mathrm{MMCD}=-\$ 1308, P<0.001)$. The reduction in all-cause medical costs $(\mathrm{MMCD}=-\$ 2251$, $P<0.001)$, which was largely driven by lower inpatient costs $(\mathrm{MMCD}=-\$ 2182, P<0.001)$ followed by lower 


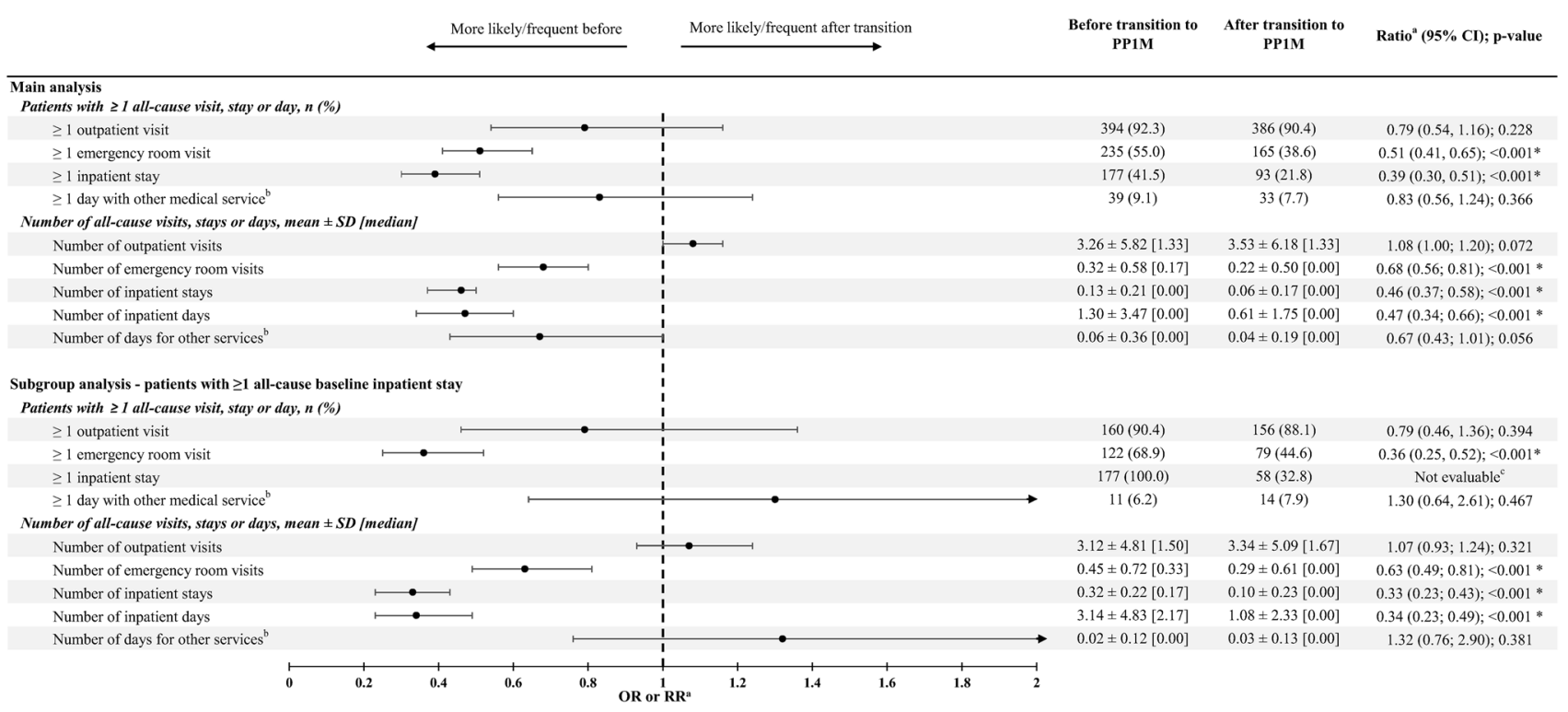

Fig. 3 Comparison of HRU 6 months before and after transition to PP1M. $C I$ confidence interval, $H R U$ healthcare resource utilization, $O R$ odds ratio, $P P 1 M$ once-monthly paliperidone palmitate, $R R$ rate ratio, $S D$ standard deviation. * Statistically significant $(P<0.05)$.
${ }^{\mathrm{a} O R s}$ were used for dichotomous variables and RRs were used for count variables. ${ }^{\mathrm{b}}$ Includes durable medical equipment, dental, and

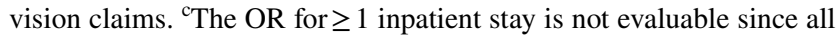
patients had an inpatient stay in the pre-PP1M transition period

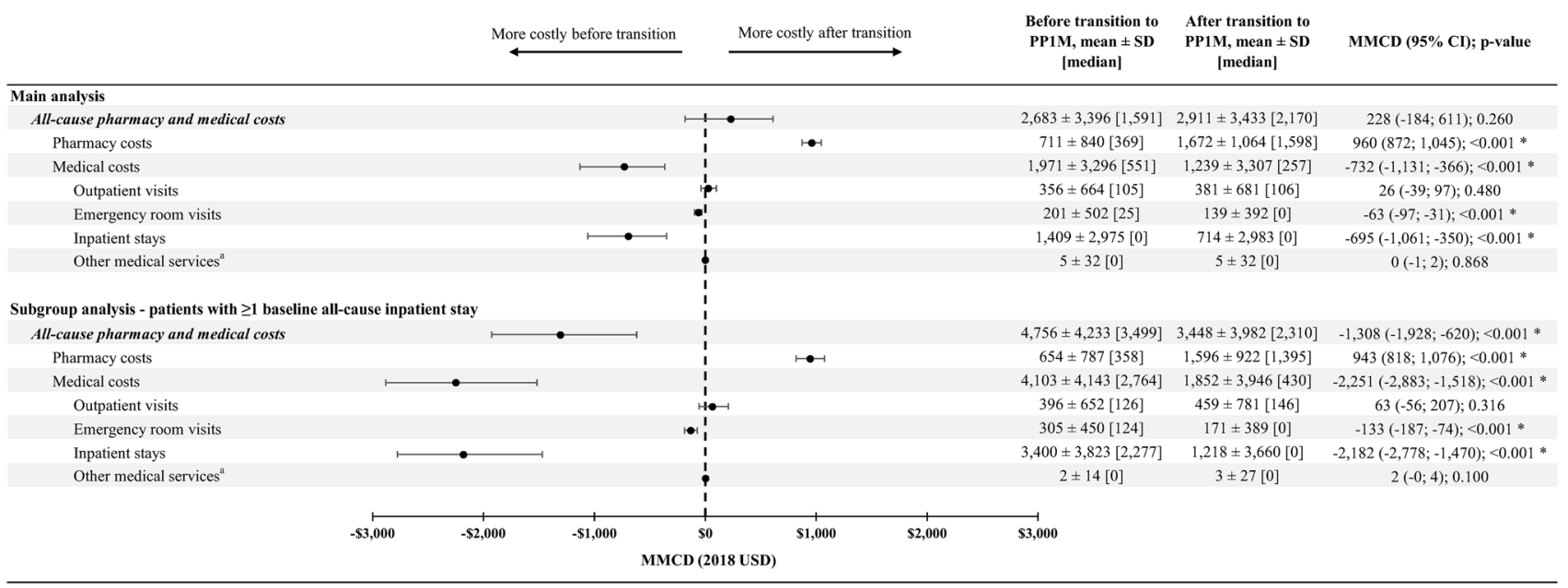

Fig. 4 Comparison of healthcare costs 6 months before and after transition to PP1M. $C I$ confidence interval; $M M C D$ mean monthly cost difference, $P P 1 M$ once-monthly paliperidone palmitate, $S D$ standard

emergency room visit costs $(\mathrm{MMCD}=-\$ 133, P<0.001)$, more than offset the increase in all-cause pharmacy costs $(\mathrm{MMCD}=\$ 943, P<0.001 ;$ Fig. 4).

\section{Discussion}

In this real-world study, patients with schizophrenia transitioning to PP1M after oral risperidone or oral paliperidone were less likely to have $\geq 1$ claim with a diagnosis of the deviation, USD US dollars. * Statistically significant $(P<0.05)$. ${ }^{\text {a In- }}$ cludes durable medical equipment, dental, and vision claims

following Elixhauser and DSM-V comorbidities: psychoses, hypertension, depression, and drug abuse, substancerelated and addictive disorders, bipolar and related disorders, sleep-wake disorders, anxiety disorders, and other conditions that may require a focus of clinical attention. Adherence to APs also improved following transition to PP1M for all patients. In terms of HRU, compared to the pre-PP1M period, patients had a fewer number of emergency room visits, inpatient stays, and days spent in an inpatient setting in the post-PP1M period. Medical costs were significantly 
reduced following transition, largely as a result of lower costs related to fewer inpatient stays and emergency room visits. Overall, total healthcare costs were similar in the preand post-PP1M transition periods, since increased pharmacy costs were offset by reduced medical costs. In the subset of patients with $\geq 1$ all-cause inpatient stay prior to PP1M transition, switching to PP1M was associated with an even greater reduction in the proportion of patients with $\geq 1$ claim for specific physical and psychiatric comorbidities, along with a similar improvement in adherence to APs and significantly lower total healthcare costs.

In contrast to prior studies that included a comparison of OAAs to PP1M using a cohort design, the current study used a pre/post design that compared patients before and after initiation of PP1M among those previously treated with oral risperidone or oral paliperidone. A strength of the pre/post design was the delineation of the benefits over time and for the same patient of switching to PP1M from an oral regimen.

Findings from this study are consistent with previous studies showing that switching from oral OAAs to LAIs could help improve adherence and outcomes by reducing dosing frequency. A large real-world cohort study of Medicaid patients with schizophrenia showed that patients initiated on second-generation LAIs had better adherence to and persistence with therapy over 12 months compared to those initiated on OAAs [17]. Similarly, in Medicaid patients recently diagnosed with schizophrenia in the US, treatment with PP1M versus OAAs was associated with better adherence, lower use of other psychiatric medications (i.e., antidepressants, anxiolytics, and mood stabilizers), and reduced medical costs resulting from fewer days spent in an inpatient setting [21]. Furthermore, reductions in inpatient and ER visits after initiating PP1M were consistent with a recent real-world study conducted in Italy in which the initiation of LAIs also resulted in reductions in these visits. Of note, the magnitude of the effect was particularly pronounced for patients who received LAIs early after disease onset [28].

In the present study, the lower likelihood of having $\geq 1$ claim with a diagnosis for hypertension post-PP1M transition, which is the most common cardiometabolic comorbidity for which patients with schizophrenia are treated [29], may reflect better adherence to hypertensive medications in addition to better adherence to APs, although this was not assessed as part of the current study. Indeed, higher adherence to APs among patients with schizophrenia has been shown to be associated with improved adherence to other medications used to manage comorbidities in these patients $[29,30]$. Moreover, the improved adherence to APs postversus pre-PP1M transition was evident despite the fact that adherence pre-PP1M transition was higher than reported elsewhere $[9,17,21]$. By design, patients were required to have at least 30 days of treatment on oral risperidone or oral paliperidone in the 60 days prior to PP1M transition to exclude treatment-naïve patients, which may explain the higher adherence rates observed in the current study relative to other studies.

A noteworthy finding of the present study was that an even greater improvement in physical and psychiatric comorbidity-related outcomes was observed in the subset of patients with $\geq 1$ all-cause inpatient stay pre-PP1M transition. Compared to the period prior to PP1M transition, total healthcare costs for these patients were lower following transition. This subset consisted of patients with a recent hospitalization and poorer overall health who were less likely to be fully adherent to treatment. These patients may particularly benefit from a switch from OAAs to PP1M, since it can help improve adherence and reduce HRU and costs. This is consistent with results from a recent 12-month decision tree model that projected a reduction in schizophrenia-related costs when switching $5 \%$ of nonadherent OAA patients to PP1M among those with a recent relapse (in the current study, having a recent hospitalization can be considered to be a proxy for recent schizophrenia relapse) [31]. Despite evidence demonstrating the efficacy and safety of LAIs, including PP1M, these medications are not as commonly used in patients with schizophrenia. This is partly due to physicians' concerns about the tolerability of LAIs and the perceived challenge of convincing patients to choose this option despite the monthly visits required for drug administration [32-34]. In addition, although there is a substantial body of evidence indicating that LAIs are associated with better efficacy than OAAs, the occurrence of some adverse events (i.e., extrapyramidal and prolactinrelated side effects) may be greater in patients treated with LAIs [16]. The present study suggests that PP1M can be considered a viable treatment alternative to oral APs. Reductions in the likelihood of claims with mental-health-related comorbidities in this study could indicate that when patients have a better control of schizophrenia symptoms, they also have a better control of other health-related complications. Patients with schizophrenia often have a high prevalence of comorbidities, including cardiometabolic abnormalities, which is a factor in treatment decisions [35-37]. The present study showed that the likelihood of claims for these comorbidities (i.e., hypertension and diabetes) was not elevated or was lower following a switch from oral risperidone or oral paliperidone to PP1M, which may imply that symptoms did not worsen following the transition.

Given that the current study only captures direct costs to payers, it is important to understand that the present analysis does not capture other dimensions of the burden of schizophrenia. For example, indirect costs resulting from productivity loss may account for at least $50 \%$ of the overall economic burden of the disease [38]. These high productivity costs can be explained by the fact that schizophrenia symptoms typically emerge during young adulthood, a period normally 
characterized by high economic productivity. Furthermore, the burden of schizophrenia also has ramifications for caregivers and families [39]. Indeed, a 2015 study conducted in Europe found that schizophrenia caregivers exhibited significantly greater absenteeism, work impairment, and associated indirect costs than matched noncaregivers [40]. Lastly, the disease also entails an intangible nonfinancial burden on patients through its symptoms and the stress and anxiety that may accompany the treatment process [39]. Achieving adequate control of schizophrenia symptoms through improved pharmacotherapy likely entails benefits affecting these other dimensions of the disease burden that go beyond the direct costs reported in the current study.

The findings of this study should be interpreted in the context of some limitations. First, claims databases may be subject to coding errors (billing inaccuracies) and missing data. Second, adherence, as measured by the PDC, was based on claims, which does not guarantee that medications were taken as prescribed. Third, costs were based on the payer's perspective, reflecting the amount paid by the insurance plan; accordingly, out-of-pocket and direct nonmedical costs paid by the patients could not be assessed. In addition, pharmacy costs did not include discounts or rebates and may have been overestimated. Therefore, the present analysis could not capture all dimensions of the burden of schizophrenia, such as societal costs and patients' quality of life. Further research is warranted to assess how switching patients from oral APs to LAIs may affect these outcomes. Fourth, the pre/post design did not allow us to adjust for factors that may change over time, since each patient acted as his or her own control. However, this limitation may be mitigated by the fact that major changes in baseline characteristics over a 12-month period are unlikely. Fifth, due to the unavailability of such data, the outcomes evaluated in the current study did not include or reflect patients' and caregivers' potential improvement in quality of life related to the switch to PP1M. Further research may be warranted to provide a more comprehensive assessment of the impact of switching to PP1M on the patients' and caregivers' quality of life. Lastly, data may not be representative of the entire US population, including patients with other types of health insurance and those without health insurance. Furthermore, only patients who received PP1M after oral risperidone or paliperidone were included per PP1M prescribing guidelines [41]. Thus, results may not be generalizable to real-world patients who received PP1M without prior treatment with oral risperidone or oral paliperidone.

\section{Conclusions}

In patients with schizophrenia in the US, transitioning to PP1M following treatment with oral risperidone or oral paliperidone was associated with a reduction in the likelihood of claims with diagnoses for specific physical and psychiatric comorbidities, an improvement in adherence to APs, and a reduction in HRU. Overall, the increase in pharmacy costs in the post-PP1M period was offset by a decrease in medical costs, suggesting that PP1M improves physical and psychiatric comorbidity-related outcomes and adherence and reduces hospitalizations and emergency room visits while remaining cost neutral. For the subset of patients with $\geq 1$ recent allcause inpatient stay prior to PP1M transition, switching to PP1M was associated with a more pronounced improvement in physical and psychiatric comorbidity-related outcomes, a similar improvement in adherence, a more pronounced reduction in HRU, and lower total healthcare costs.

Acknowledgements Medical writing assistance was provided by Sara Kaffashian, an employee of Analysis Group, Inc.

Author Contributions All authors contributed to the study design. Data collection and analysis were performed by BE, MHL, ACS, and PL. All authors contributed to the interpretation of results, manuscript preparation, and manuscript review and editing.

Data Availability Statement The datasets generated and analyzed during the current study are not publicly available because they were used pursuant to a data use agreement. The data are available through requests made directly to IQVIA.

\section{Compliance with Ethical Standards}

Funding This work was supported by Janssen Scientific Affairs, LLC. The sponsor was involved in the study design, interpretation of results, manuscript preparation, and publication decisions.

Conflict of interest BE, MHL, ACS, and PL are employees of Analysis Group, Inc., a consulting company that has provided paid consulting services to Janssen Scientific Affairs, LLC, which funded the development and conduct of this study and manuscript. CP is an employee of Johnson \& Johnson and NT and ACEK are employees and stockholders of Johnson \& Johnson.

Open Access This article is distributed under the terms of the Creative Commons Attribution-NonCommercial 4.0 International License (http://creativecommons.org/licenses/by-nc/4.0/), which permits any noncommercial use, distribution, and reproduction in any medium, provided you give appropriate credit to the original author(s) and the source, provide a link to the Creative Commons license, and indicate if changes were made.

\section{References}

1. Tandon R, Nasrallah HA, Keshavan MS. Schizophrenia, “just the facts" 4. Clinical features and conceptualization. Schizophr Res. 2009;110(1-3):1-23. https://doi.org/10.1016/j.schre s.2009.03.005.

2. Leucht S, Arbter D, Engel RR, Kissling W, Davis JM. How effective are second-generation antipsychotic drugs? A meta-analysis of placebo-controlled trials. Mol Psychiatry. 2009;14(4):429-47. https://doi.org/10.1038/sj.mp.4002136. 
3. Leucht S, Tardy M, Komossa K, Heres S, Kissling W, Salanti G, et al. Antipsychotic drugs versus placebo for relapse prevention in schizophrenia: a systematic review and meta-analysis. Lancet. 2012;379(9831):2063-71. https://doi.org/10.1016/S0140 $-6736(12) 60239-6$.

4. Correll CU, Citrome L, Haddad PM, Lauriello J, Olfson M, Calloway SM, et al. The use of long-acting injectable antipsychotics in schizophrenia: evaluating the evidence. J Clin Psychiatry. 2016;77(suppl 3):1-24. https://doi.org/10.4088/JCP.15032su1.

5. Marcus SC, Zummo J, Pettit AR, Stoddard J, Doshi JA. Antipsychotic adherence and rehospitalization in schizophrenia patients receiving oral versus long-acting injectable antipsychotics following hospital discharge. J Manag Care Spec Pharm. 2015;21(9):754-68. https://doi.org/10.18553/jmcp.2015.21.9.754.

6. Lang K, Federico V, Muser E, Menzin J, Menzin J. Rates and predictors of antipsychotic non-adherence and hospitalization in Medicaid and commercially-insured patients with schizophrenia. J Med Econ. 2013;16(8):997-1006. https://doi.org/10.3111/13696 998.2013.816310.

7. Ascher-Svanum H, Faries DE, Zhu B, Ernst FR, Swartz MS, Swanson JW. Medication adherence and long-term functional outcomes in the treatment of schizophrenia in usual care. J Clin Psychiatry. 2006;67(3):453-60.

8. Haddad PM, Brain C, Scott J. Nonadherence with antipsychotic medication in schizophrenia: challenges and management strategies. Patient Relat Outcome Meas. 2014;5:43-62. https://doi. org/10.2147/PROM.S42735.

9. MacEwan JP, Forma FM, Shafrin J, Hatch A, Lakdawalla DN, Lindenmayer JP. Patterns of adherence to oral atypical antipsychotics among patients diagnosed with schizophrenia. J Manag Care Spec Pharm. 2016;22(11):1349-61. https://doi.org/10.18553 /jmcp.2016.22.11.1349.

10. Novick D, Haro JM, Suarez D, Perez V, Dittmann RW, Haddad PM. Predictors and clinical consequences of non-adherence with antipsychotic medication in the outpatient treatment of schizophrenia. Psychiatry Res. 2010;176(2-3):109-13. https://doi. org/10.1016/j.psychres.2009.05.004.

11. Sun SX, Liu GG, Christensen DB, Fu AZ. Review and analysis of hospitalization costs associated with antipsychotic nonadherence in the treatment of schizophrenia in the United States. Curr Med Res Opin. 2007;23(10):2305-12. https://doi.org/10.1185/03007 $9907 \times 226050$.

12. Dilla T, Ciudad A, Alvarez M. Systematic review of the economic aspects of nonadherence to antipsychotic medication in patients with schizophrenia. Patient Prefer Adherence. 2013;7:275-84. https://doi.org/10.2147/PPA.S41609.

13. Marcus SC, Olfson M. Outpatient antipsychotic treatment and inpatient costs of schizophrenia. Schizophr Bull. 2008;34(1):17380. https://doi.org/10.1093/schbul/sbm061.

14. Liu J. Balancing therapeutic safety and efficacy to improve clinical and economic outcomes in schizophrenia: a managed care perspective. Am J Manag Care. 2014;20(8 Suppl):S174-83.

15. Kaplan G, Casoy J, Zummo J. Impact of long-acting injectable antipsychotics on medication adherence and clinical, functional, and economic outcomes of schizophrenia. Patient Prefer Adherence. 2013;7:1171-80. https://doi.org/10.2147/PPA.S53795.

16. Park SC, Choi MY, Choi J, Park E, Tchoe HJ, Suh JK, et al. Comparative efficacy and safety of long-acting injectable and oral second-generation antipsychotics for the treatment of schizophrenia: a systematic review and meta-analysis. Clin Psychopharmacol Neurosci. 2018;16(4):361-75. https://doi. org/10.9758/cpn.2018.16.4.361.

17. Pilon D, Tandon N, Lafeuille MH, Kamstra R, Emond B, Lefebvre $\mathrm{P}$, et al. Treatment patterns, health care resource utilization, and spending in medicaid beneficiaries initiating second-generation long-acting injectable agents versus oral atypical antipsychotics. Clin Ther. 2017;39(10):1972. https:// doi.org/10.1016/j.clinthera.2017.08.008.

18. Emsley R, Kilian S. Efficacy and safety profile of paliperidone palmitate injections in the management of patients with schizophrenia: an evidence-based review. Neuropsychiatr Dis Treat. 2018;14:205-23. https://doi.org/10.2147/ndt.s139633.

19. Nussbaum AM, Stroup TS. Paliperidone palmitate for schizophrenia. Cochrane Database Syst Rev. 2012(6):Cd008296. https ://doi.org/10.1002/14651858.cd008296.pub2.

20. Janssen. Invega Sustenna-highlights of prescribing information 2018. http://www.janssenlabels.com/package-insert/produ ct-monograph/prescribing-information/INVEGA+SUSTENNApi.pdf. Accessed 13 Dec 2018.

21. Pilon D, Muser E, Lefebvre P, Kamstra R, Emond B, Joshi $K$. Adherence, healthcare resource utilization and Medicaid spending associated with once-monthly paliperidone palmitate versus oral atypical antipsychotic treatment among adults recently diagnosed with schizophrenia. BMC Psychiatry. 2017;17(1):207. https://doi.org/10.1186/s12888-017-1358-3.

22. Muser E, Kozma CM, Benson CJ, Mao L, Starr HL, Alphs L, et al. Cost effectiveness of paliperidone palmitate versus oral antipsychotics in patients with schizophrenia and a history of criminal justice involvement. J Med Econ. 2015;18(8):637-45. https://doi.org/10.3111/13696998.2015.1037307.

23. Manjelievskaia J, Amos TB, El Khoury AC, Vlahiotis A, Cole A, Juneau P. A comparison of treatment patterns, healthcare resource utilization, and costs among young adult Medicaid beneficiaries with schizophrenia treated with paliperidone palmitate or oral atypical antipsychotics in the US. J Med Econ. 2018;21(12):1221-9. https://doi.org/10.1080/13696 998.2018.1527608.

24. US Department of Health and Human Services. 45 CFR 46. https ://www.hhs.gov/ohrp/regulations-and-policy/regulations/45cfr-46/index.html\#46.101. Accessed 05 Nov 2019.

25. Elixhauser A, Steiner C, Kruzikas D. Comorbidity software documentation. HCUP Methods Series, report number 2004-1. Rockville, MD: Agency for Healthcare Research and Quality; 2004. http://www.hcup-us.ahrq.gov/reports/ComorbiditySoftwareD ocumentationFinal.pdf. Accessed 05 Dec 2018.

26. American Psychiatric Association. Diagnostic and statistical manual of mental disorders: DSM-V. Washington, DC: American Psychiatric Publ. Inc.; 2013.

27. Bureau of Labor Statistics. Consumer Price Index. https://www. bls.gov/cpi/tables/supplemental-files/home.htm. Accessed 15 Feb 2019.

28. Poloni N, Ielmini M, Caselli I, Lucca G, Gasparini A, Gasparini A, et al. Oral antipsychotic versus long-acting injections antipsychotic in schizophrenia spectrum disorder: a mirror analysis in a real-world clinical setting. Psychopharmacol Bull. 2019;49(2):17-27.

29. Hansen RA, Maciejewski M, Yu-Isenberg K, Farley JF. Adherence to antipsychotics and cardiometabolic medication: association with health care utilization and costs. Psychiatr Serv. 2012;63(9):920-8. https://doi.org/10.1176/appi.ps.201100328.

30. Lafeuille M-H, Tandon N, Tiggelaar S, Kamstra R, Lefebvre P, Kim E, et al. Economic impact in Medicaid beneficiaries with schizophrenia and cardiometabolic comorbidities treated with once-monthly paliperidone palmitate vs. oral atypical antipsychotics. Drugs Real World Outcomes. 2018;5(1):81-90. https:// doi.org/10.1007/s40801-018-0130-4.

31. El Khoury AC, Pilon D, Morrison L, Shak N, Vermette-Laforme $\mathrm{M}, \mathrm{Amos} \mathrm{TB}$, et al. The prospective economic impact of once monthly paliperidone palmitate versus oral atypical antipsychotics in Medicaid patients with schizophrenia. Curr Med Res Opin. 2019;35(3):395-405. https://doi.org/10.1080/03007 995.2018.1558195. 
32. Bossie CA, Fu DJ, Sliwa JK, Ma YW, Alphs L. Tolerability of initiation doses of once-monthly paliperidone palmitate in patients with recently diagnosed schizophrenia in an acute treatment trial. Ther Adv Psychopharmacol. 2011;1(4):111-24. https://doi. org/10.1177/2045125311413006.

33. Heres S, Lambert M, Vauth R. Treatment of early episode in patients with schizophrenia: the role of long acting antipsychotics. Eur Psychiatry J Assoc Eur Psychiatr. 2014;29(Suppl 2):1409-13. https://doi.org/10.1016/s0924-9338(14)70001-x.

34. Manchanda R, Chue P, Malla A, Tibbo P, Roy MA, Williams R, et al. Long-acting injectable antipsychotics: evidence of effectiveness and use. Can J Psychiatry. 2013;58(5 Suppl 1):5s-13s.

35. Bresee LC, Majumdar SR, Patten SB, Johnson JA. Prevalence of cardiovascular risk factors and disease in people with schizophrenia: a population-based study. Schizophr Res. 2010;117(1):75-82. https://doi.org/10.1016/j.schres.2009.12.016.

36. Gardner-Sood P, Lally J, Smith S, Atakan Z, Ismail K, Greenwood KE, et al. Cardiovascular risk factors and metabolic syndrome in people with established psychotic illnesses: baseline data from the IMPaCT randomized controlled trial. Psychol Med. 2015;45(12):2619-29. https://doi.org/10.1017/s00332917150005 62.
37. Mitchell AJ, Vancampfort D, Sweers K, van Winkel R, Yu W, De Hert M. Prevalence of metabolic syndrome and metabolic abnormalities in schizophrenia and related disorders-a systematic review and meta-analysis. Schizophr Bull. 2013;39(2):306-18. https://doi.org/10.1093/schbul/sbr148.

38. Chong HY, Teoh SL, Wu DB, Kotirum S, Chiou CF, Chaiyakunapruk N. Global economic burden of schizophrenia: a systematic review. Neuropsychiatr Dis Treat. 2016;12:357-73. https://doi. org/10.2147/NDT.S96649.

39. Tajima-Pozo K, de Castro Oller MJ, Lewczuk A, Montanes-Rada F. Understanding the direct and indirect costs of patients with schizophrenia. F1000Res. 2015;4:182. https://doi.org/10.12688 /f1000research.6699.2.

40. Gupta S, Isherwood G, Jones K, Van Impe K. Productivity loss and resource utilization, and associated indirect and direct costs in individuals providing care for adults with schizophrenia in the EU5. Clinicoecon Outcomes Res. 2015;7:593-602. https://doi. org/10.2147/CEOR.S94334.

41. US Food and Drug Administration. Prescribing informationInvega Sustenna ${ }^{\circledR}$ (paliperidone palmitate). 2018. https://www. accessdata.fda.gov/drugsatfda_docs/label/2018/022264s027lbl. pdf. Accessed 08 Aug 2018.

\section{Affiliations}

\section{Charmi Patel $^{1} \cdot$ Bruno Emond $^{2} \cdot$ Marie-Hélène Lafeuille $^{2} \cdot$ Aurélie Côté-Sergent $^{2} \cdot$ Patrick Lefebvre $^{2} \cdot$ Neeta Tandon $^{1}$. Antoine C. El Khoury ${ }^{1}$}

Charmi Patel

cpate134@its.jnj.com

Bruno Emond

bruno.emond@analysisgroup.com

Marie-Hélène Lafeuille

marie-helene.lafeuille@ analysisgroup.com

Aurélie Côté-Sergent

aurelie.cote-sergent@ analysisgroup.com

Patrick Lefebvre

patrick.lefebvre@analysisgroup.com
Neeta Tandon

ntandon@its.jnj.com

Antoine C. El Khoury

akhour@its.jnj.com

Janssen Scientific Affairs, LLC., 1125 Trenton-Harbourton Road, Titusville, NJ 08560, USA

2 Analysis Group, Inc., 1190 Avenue des Canadiens-de-Montreal, Tour Deloitte, Suite 1500, Montreal, QC H3B 0G7, Canada 